\title{
EL ROL DE LA ARGUMENTACIÓN EN EL PENSAMIENTO CRÍTICO Y EN LA ESCRITURA EPISTÉMICA EN BIOLOGÍA E HISTORIA: APROXIMACIÓN A PARTIR DE LAS REPRESENTACIONES SOCIALES DE LOS DOCENTES ${ }^{1}$
}

The role of argumentation in critical thinking and epistemic writing in Biology and History: An approach from the social representations of teachers

\author{
Alejandro Córdova Jiménez* \\ Marisol Velásquez Rivera** \\ Lisbeth Arenas Witker***
}

Resumen

El desarrollo de la argumentación en el proceso de alfabetización académica cobra especial importancia debido a que el conocimiento académico es esencialmente argumentativo (Serrano y Villalobos, Niveles discursivos). Por esto, el objetivo de esta investigación es, por un lado, relevar, a partir del discurso de los docentes en un programa de Biología e Historia, las representaciones sociales acerca de la enseñanza-aprendizaje de la argumentación y, por otro, generar un modelo explicativo de este fenómeno. La recolección de datos se realizó por medio de entrevistas a docentes de ambas disciplinas y el análisis de ellos, mediante la técnica de análisis de contenido, la que busca formular inferencias reproducibles y válidas que puedan aplicarse a un contexto determinado (Krippendorff, 1997).

Palabras clave: Argumentación. Escritura académica. Representaciones sociales. Licenciatura en Biología. Licenciatura en Historia.

\begin{abstract}
The development of argumentation in the process of academic literacy is particularly important because scientific knowledge is essentially argumentative (Serrano y Villalobos, Niveles discursivos). For this reason, the purpose of this research is to elicit social representations about teaching and learning of argumentation from the discourse of teachers in a program of Biology and History, as well as to generate an explanatory model for this phenomenon. The data collection instrument used was an interview to teachers in both disciplines, and the data was analyzed using content analysis, a technique that seeks to develop replicable and valid inference that can be applied to a given context (Krippendorff, 1997).
\end{abstract}

Key words: Argumentation, Academic writing, Social representations, Bachelor's degree in Biology, Bachelor's degree in History

\footnotetext{
1 Trabajo financiado y desarrollado en el marco del Proyecto FONDECYT 1120549: "Alfabetización académica: escritura epistémica y argumentación en ciencias naturales y sociales en la Universidad".
} 


\section{INTRODUCCIÓN}

La alfabetización académica es un tema que se ha convertido en foco de interés desde la última década en el mundo hispanohablante (Carlino). Esto se evidencia, por ejemplo, en la gran cantidad de cursos o talleres de comprensión o redacción que se imparten al comienzo de los estudios superiores, cuya finalidad es enseñar a leer y escribir a los estudiantes universitarios como una manera de ayudarlos en su proceso de inserción disciplinar y evitar la reprobación de asignaturas (Fernández; Carlino; Carlino, Iglesias y Laxalt), lo que provoca, en muchas ocasiones, altos índices de deserción académica. Numerosas son las experiencias que pueden recogerse en las universidades latinoamericanas en que se desarrollan actividades que están orientadas a: a) enseñar a escribir y leer como lo hacen los especialistas, b) enseñar a leer y a escribir para apropiarse del conocimiento producido por la comunidad disciplinar y c) enseñar a leer y a escribir para generar nuevo conocimiento (Carlino, 359), todo lo anterior considerando el potencial epistémico de los procesos y prácticas de lectura y escritura.

Por tanto, conseguir que los nuevos miembros de las distintas comunidades desarrollen una escritura epistémica es fundamental. En términos de Carlino, la escritura es un instrumento que permite desarrollar, revisar y transformar el conocimiento adquirido, lo que posibilita al estudiante apropiarse del conocimiento de la comunidad $\mathrm{y}$, a su vez, transformarlo. Al respecto, Marinkovich, Velásquez y Olivares señalan que "alcanzar el nivel epistémico en escritura significa una nueva forma de pensar que implica, a su vez, transformaciones cognitivas no solo en los individuos, sino también en las comunidades y en la sociedad en la que estos están insertos" (131).

Para conseguir este nivel epistémico los estudiantes universitarios deben previamente haber desarrollado ciertas habilidades de pensamiento crítico: analizar, comparar, evaluar argumentos y posturas, jerarquizar, contrastar, categorizar, discutir, etcétera, y la argumentación es la instancia perfecta para que estas se potencien.

A partir de esta relación que pudiera parecer -a primera impresión- lineal, se hace necesario profundizar en la argumentación como actividad cognitiva que permite el desarrollo del pensamiento crítico y, más adelante, la escritura epistémica. Si en las aulas universitarias se considera la argumentación como núcleo central de los procesos de aprendizaje de los estudiantes, es posible suponer que consecuentemente se desarrollarán, por ejemplo, habilidades de evaluación de posturas, juicios u opiniones, lo que se constituirá como un paso esencial en la objetivación del conocimiento disciplinar, condición previa de la escritura epistémica. Obviamente, su afianzamiento en la universidad posibilitará un desarrollo cognitivo más elaborado y complejo, que redundará en que las habilidades de pensamiento se refinarán y, por tal razón, también la argumentación. Así, el círculo se irá enriqueciendo.

Frente a este panorama resulta importante preguntarse ¿cómo se representan la argumentación los docentes universitarios?, ¿qué relaciones establecen entre la argumentación, los procesos cognitivos y la escritura?, ¿cómo conciben la enseñanza de la argumentación en las aulas universitarias?, etcétera. 
A partir de las preguntas generadoras anteriores, los objetivos que se persiguen con este trabajo son: relevar, a partir del discurso de los docentes de un programa de Biología e Historia, las representaciones sociales (RS) acerca de la argumentación, generando un modelo explicativo en cada una de las disciplinas.

Para ello se presentará, en primer lugar, el concepto de argumentación y la relación que tiene con la alfabetización académica; en segundo lugar, la argumentación en relación con el pensamiento crítico y, finalmente, la argumentación con la escritura epistémica. Teniendo claro dichos conceptos y las relaciones que entre ellos se pueden establecer, se presenta la metodología de este artículo y los resultados obtenidos en cada una de las disciplinas.

\section{MARCO TEÓRICO}

\subsection{LA ARGUMENTACIÓN Y SU RELACIÓN CON LA ALFABETIZACIÓN ACADÉMICA}

La argumentación se concibe como una práctica discursiva cuya función comunicativa se centra en dirigirse al receptor para lograr su adhesión frente a la idea que se plantea (Toulmin; Van Eemeren, Grootendorst y Snoeck; Perelman y Olbrecht-Tyteca; Gorodokin y Mercau) y está constituida por un conjunto de razonamientos en torno a problemas en que el individuo evalúa las ideas, creencias y opiniones (llardo; Otis).

De esta manera, la argumentación permite evidenciar la expresión del punto de vista de un individuo, el que apoyará su postura con el uso de argumentos (a favor o en contra) utilizando distintos razonamientos. Cobra un rol esencial en ámbitos universitarios, debido a que el conocimiento científico es inminentemente argumentativo, así como lo señalan Serrano y Villalobos:

la mayoría de los textos académicos en que se expresa [la argumentación], contiene explicaciones, conceptos, principios y métodos, sobre los cuales es necesario identificar la postura del autor y las posiciones que se mencionan de otros autores, tales como reconocer y justificar las controversias de las que han emergido y tomar posturas frente al conocimiento. Para todo esto se requiere saber argumentar y así poder integrarse a las prácticas académicas, inherentes al dominio de la cultura de las disciplinas vinculadas a la comunidad científica o profesional (La argumentación, 152).

En el caso de las disciplinas que se consideran al interior de las humanidades, son fundamentales las interpretaciones personales explícitas, por lo que la argumentación resulta de una importancia decisiva. Por tanto, la utilización de recursos para expresar puntos de vista, compararlos, discutirlos y defenderlos es mucho más común que en otras áreas (Navarro).

Sin lugar a dudas, al señalar la importancia que posee la argumentación para lograr eficazmente el proceso de alfabetización académica cabe cuestionarse si efectivamente se consigue. Evidentemente, muchas veces los estudiantes ingresan al mundo universitario con dificultades en las habilidades discursivas académicas, 
comprendiendo escasamente, con vago desarrollo de la expresión oral y grandes dificultades en la escritura (Padilla, Douglas y López).

A su vez, es posible evidenciar la agudización de esta problemática por la preferencia escolarizada del aprendizaje reproductivo y memorístico, condicionando a los estudiantes a pensar que el conocimiento es estable y poco cuestionable. Esto desplaza todo desarrollo de habilidades más complejas como la argumentación, situación que se evidencia en todos los niveles de formación académica (Padilla, Producción; Taller; Iniciación). No obstante, esta práctica, en muchas ocasiones, se refuerza en la Universidad, evidenciando la evaluación de la reproducción del conocimiento que otro señaló, su memorización y transmisión tal y como está escrito, sin entregar la posibilidad de que se profundice en el mismo y en su construcción. Así, se condiciona a realizar lecturas pasivas y reproducir en desmedro de realizar lecturas argumentativas para ir generando nuevos y más sólidos conocimientos (Padilla, Douglas y López).

A partir de esto, la relación que se establece entre la argumentación y la alfabetización académica es innegable. Si bien la primera es parte de la segunda, también es cierto que se nutren, se configuran y se interrelacionan, pues ambas se van construyendo y reconstruyendo a partir de un proceso que pone en relación al sujeto aprendiente con la disciplina.

\subsection{LA ARGUMENTACIÓN Y EL PENSAMIENTO CRÍTICO}

La argumentación, como antes ya se señaló, tiene como propósito fundamental dar cuenta de la opinión que se tiene a partir de un punto de vista, mediante argumentos razonados que sostienen la postura planteada. Constituye el punto de partida ideal para desarrollar distintas habilidades de pensamiento crítico, como poseer una claridad de planteamientos, identificar puntos de vista ambiguos, evaluar los argumentos, entre otros (Mota de Cabrera). Obviamente, antes de evaluar los argumentos ajenos es necesario ser capaz de argumentar las ideas y posturas propias, entonces la relación que se establece entre ambos constructos no es unidireccional, sino más bien de interdependencia.

Para autores como Fisher y Scriven el pensamiento crítico se encuentra ligado a los procesos que se realizan al leer y escribir en los ámbitos académicos, señalando que su definición estaría en "la activa y efectiva interpretación de observaciones, comunicaciones, información y argumentación" (21), mientras que Richard Paul y Linda Elder lo reconocen como una forma de pensamiento acerca de cualquier problema o tema en que el individuo sea capaz de afinar y mejorar sus ideas, apropiándose de estructuras esenciales al pensamiento, transformándolas en patrones intelectuales. Es así como el pensador crítico sería capaz de evidenciar razonamientos mal construidos, fallas, evaluar diferentes puntos de vista, evidenciar inferencias, entre otras cosas, lo que hace fundamental que su desarrollo se encuentre en la formación disciplinar (Mota de Cabrera). 
A partir de esto, la argumentación se lleva a cabo cuando se ponen en juego todas las habilidades de pensamiento crítico, al construir un argumento o al evaluarlo; al sostener una postura previamente, es imprescindible que el sujeto sea capaz de analizar los antecedentes, comprenderlos, dar cuenta de las inferencias realizadas, entre otras cosas. Sin la presencia de las habilidades esenciales del pensamiento crítico, la argumentación no puede consolidarse, pero al mismo tiempo, sin las instancias para poner en juego la argumentación no es posible desarrollar las habilidades de pensamiento crítico. De este modo, se produce una sinergia entre ambos constructos.

\subsection{ARGUMENTACIÓN Y ESCRITURA EPISTÉMICA}

La educación universitaria no está solamente enfocada a que los sujetos que ingresan aprendan ciertos contenidos que les permitan obtener un grado o un título profesional, sino que también trae aparejado un proceso de enculturación de las prácticas académicas que circulan en las diferentes comunidades. De este modo, apropiarse de la lectura y de la escritura en la Universidad es un proceso complejo, sobre todo porque están determinadas por la naturaleza del conocimiento y por los modos distintos de acercarse a él que se evidencian en cada una de las comunidades (Navarro).

Escribir en la educación universitaria es una tarea ardua, especialmente porque requiere que el sujeto proponga ideas, analice, plantee, critique y ponga en perspectiva las evidencias propias de su disciplina; asimismo, debe tener presente que en cada comunidad se reconocen ideas a veces contradictorias unas con otras y que son objeto de discusión al interior de la misma comunidad. En este contexto, la escritura epistémica, como actividad permanente y generadora de aprendizajes, debiera ser prioritaria en la formación universitaria, pues permite la generación de nuevo conocimiento y debiera considerar el desarrollo de un componente argumentativo, desde los primeros años de ingreso a las aulas universitarias.

En síntesis, la argumentación posee un rol fundamental en el acceso al conocimiento disciplinar y en la transformación del mismo, ello permite el satisfactorio proceso de alfabetización académica. Para que la argumentación y el desarrollo de las habilidades de pensamiento crítico se lleven a cabo se requiere de la orquestación de ambos en un constructo disciplinar que los articule en una progresión en que el rol del especialista es fundamental. Las relaciones entre argumentación, habilidades de pensamiento crítico y escritura epistémica se presentan en la siguiente figura: 


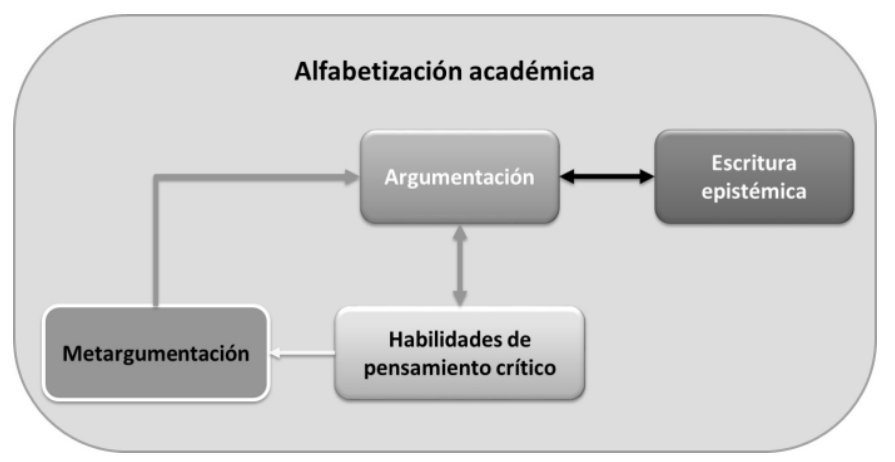

Figura 1: Esquema de relación de argumentación, habilidades de pensamiento crítico y escritura epistémica

La argumentación como eje central del modelo permite el desarrollo de habilidades de pensamiento crítico y estas, a su vez, contribuyen a su desarrollo. Ambas permiten que el sujeto pueda llevar su propia argumentación a la conciencia y regularla (metaargumentación) y esta, a su vez, nutre a la argumentación y, por ende, a la escritura epistémica.

\section{MetOdOLOGÍA}

\subsection{REPRESENTACIONES SOCIALES}

En esta investigación las RS se entenderán como una creencia u opinión acerca de un tema determinado e identificable en el discurso de los docentes, es decir, en ningún caso es la realidad absoluta sino que la mirada o visión que exteriorizan por medio del lenguaje, la forma en que reconstruyen esa porción de realidad. Asimismo, se considerarán aquellas ideas o expresiones que se reiteran en el discurso, esto debido al carácter social que contiene una RS, sin confundirla con alguna idea personal o propia que pueda tener un sujeto frente al tema en cuestión. Por tanto, las recurrencias en los discursos serán los contenidos de estas RS (Moscovici; Jodelet).

\subsection{TIPO DE INVESTIGACIÓN Y SU ENFOQUE}

El tipo de investigación es de corte cualitativo y se basa en un enfoque ascendente (bottom up). La investigación cualitativa puede ser entendida como un intento de comprensión global de un fenómeno o un objeto de estudio, es por ello que el contenido de ese objeto es abordado en su totalidad. A partir de este diseño metodológico, el enfoque bottom up parte de los datos para que, a partir de ellos, se levanten categorías que permitirán establecer ciertas regularidades que podrían conformar teorías emergentes. 


\subsection{MUESTRA}

La muestra que se seleccionó para la realización de esta investigación son seis entrevistas en profundidad de tipo semiestructuradas a docentes de un programa de Licenciatura en Biología y seis entrevistas a docentes de un programa de Licenciatura en Historia, ambos pertenecen a una Universidad del H. Consejo de Rectores de Chile.

\subsection{TÉCNICA DE ANÁLISIS}

La técnica que se utilizó para el análisis de los datos es el análisis de contenido. Esta permite investigar los discursos y su naturaleza, es decir, analizar detalladamente cualquier tipo de comunicación humana de manera objetiva, sistemática, cualitativa y cuantitativa, permitiendo obtener generalizaciones (Holsti; Porta y Silva). Asimismo, se realizan diversos pasos en el análisis de los datos, con el fin de alcanzar la etapa de interpretación.

En esta investigación se desarrollaron los siguientes pasos: a) determinación de los objetivos de la investigación; b) establecimiento y definición de las unidades de análisis (unidades de contexto y unidad de registro); c) establecimiento y definición de las categorías, a saber, categorías preliminares $(\mathrm{CP})$ y, en su agrupación por relaciones de sentido, las categorías generales (CG), de naturaleza más inclusiva y global; d) codificación tras el relevamiento de las $\mathrm{CP}$ y las $\mathrm{CG}$, asignando etiquetas que contribuirán a facilitar el análisis y la presentación de los resultados; e) establecimiento de frecuencia de las mismas y, finalmente, f) interpretación cualitativa de los datos y consolidación teórica, intentando dar respuestas a las preguntas de investigación y a los objetivos. Se obtiene así la construcción de un modelo explicativo que dé cuenta del fenómeno estudiado.

\section{ANÁLISIS Y DISCUSIÓN DE LOS RESULTADOS}

\subsection{El CASO DE LA LICENCIATURA EN BIOLOGÍA}

El análisis de las seis entrevistas en profundidad a los docentes del Programa de Licenciatura en Biología arrojó $481 \mathrm{CP}$, que fueron agrupadas en $12 \mathrm{CG}$, a saber, Evaluación, Géneros, Escritura en Ciencias, Alfabetización académica, Rol docente, Estrategias metodológicas, Habilidades necesarias en Ciencias, Clasificación de los estudiantes, Dificultades de los estudiantes, Argumentación, Pensamiento crítico y Artefactos multimodales. Si bien se releva una categoría específica para Argumentación -que constituye $5,4 \%$ del total de los datos- otras seis están asociadas a concepciones ligadas a esta. Por ejemplo, en la CG Habilidades necesarias en Ciencias se considera una CP denominada Dificultades al pensar/rebatir y otra designada como Dificultades para redactar el apartado Discusión. Resulta evidente que las CP anteriores están concebidas en relación con la Argumentación, pues rebatir implica poner en discusión una idea o punto de vista al que no se adhiere; lo mismo ocurre con el apartado 
Discusión, pues este implica - a juicio de la comunidad- la defensa de los resultados obtenidos en una investigación de carácter científico. De este modo, de las 481 CP, 96 están asociadas -en mayor o menor medida- a la categoría Argumentación, lo que alcanza $19,96 \%$

A continuación se presenta el Gráfico 1 en que se detalla el porcentaje de cada una de las CG que se relevaron del discurso de los docentes.

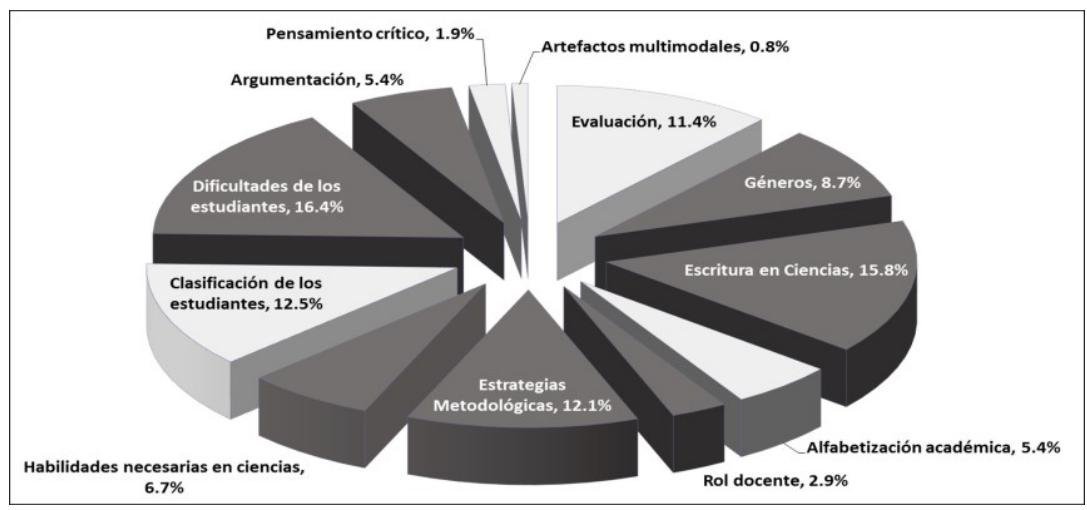

Gráfico 1: Categorías generales (CG) del discurso de docentes en Biología

A partir del gráfico anterior y siguiendo la técnica de análisis de contenido, se procede a elaborar un modelo explicativo que tiene como centro la Argumentación y que la relaciona con las habilidades de pensamiento crítico y con la escritura epistémica.

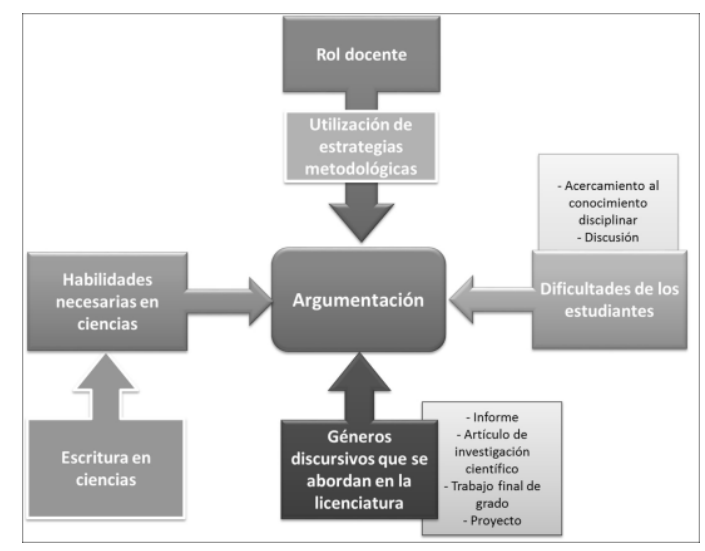

Figura 2: Modelo explicativo de Biología

Una de las primeras consideraciones que se deben tomar en cuenta es la estrecha relación que los docentes atribuyen a los géneros discursivos que reconocen que 
circulan en la disciplina (informes de salida a terreno, de laboratorio, artículos de investigación, paper de estudiantes, trabajos finales de grado y proyectos) con la Argumentación en Biología, pues se constituye como una de las principales habilidades en un contexto científico y se suele utilizar como una forma de justificar la información presentada. Esta Argumentación se ampara en el conocimiento disciplinar, por lo que todos los hallazgos que se describan en cualquier texto deben considerar y discutirse con los conocimientos que se han consensuado previamente en la comunidad, aun cuando tratándose de un contexto académico las exigencias para los estudiantes no sean las mismas que para los especialistas:

Entonces, lo que ellos tienen que hacer es buscar, no como uno lo hace generalmente en un proyecto de verdad -nuestra bibliografía es escasa en español- y basado en esa bibliografia, ellos creen todo eso que están leyendo y ahí se transforman en ese experto y de ahí es de donde ellos tienen los argumentos para justificar (BIO6).

Un aspecto que se debe considerar es que los docentes declaran abiertamente que no se hacen cargo de enseñar a escribir, lo que impactaría, por un lado, evidentemente en la forma en cómo los estudiantes aprenden a escribir estos géneros y, por el otro, en la manera cómo aprenden a argumentar en la disciplina. Sobre esto último, debe considerarse, como señala Teberosky, que el propósito mismo de la escritura en el ámbito científico es esencialmente argumentativo, ya que se busca que el nuevo conocimiento generado pueda ser integrado a los saberes del campo disciplinar en que se desarrolla, por lo que debe ser previamente aprobado y validado por la comunidad científica en cuestión.

Este reparo en abordar la enseñanza de la escritura puede estar asociado con falta de tiempo y/o no tener las herramientas para hacerlo por no ser parte del dominio disciplinar, así como lo señalan (Arenas y Olivares; Córdova y Velásquez).

Yo no soy especialista en argumentación, pero he leído ciertas cosas, pero no lo aplico [...] Yo no sé enseñarla, me gustaría saber enseñar a argumentar bien [...], yo lo hago de manera natural, pero no lo sé enseñar, ni tengo tiempo para hacerlo (BIO2).

En menor medida, indican que su enseñanza no corresponde a una obligación que les competa:

No nos corresponde tampoco hacer estas cosas de lenguaje... creo que es responsabilidad de la universidad que debería tomar estas cosas y creo que esto debería ser fuera del currículum [...] el chico si quiere lo hace fuera, es problema de él. Y la universidad debería responsabilizarse de eso y otras cosas como esta del lenguaje, porque uno... o sea uno sabe del colegio (BIO1).

Entonces, frente a esto, es posible preguntarse ¿cómo los estudiantes aprenden a argumentar en la disciplina? Del discurso de los docentes se desprende que utilizan 
ciertas estrategias metodológicas para apoyar el proceso de aprendizaje: mecánicas de discusión en clases o promoción de la lectura de textos modelos sobre los cuales los estudiantes producen los propios. Este tipo de ejercicios han sido introducidos para reparar las dificultades que reconocen al momento de evaluar los escritos de sus estudiantes, pues, de lo contrario, no conseguirían cumplir con las exigencias para incorporarse en la comunidad disciplinar al egresar del programa de estudios.

Los docentes reconocen la dificultad de los estudiantes para acercarse y apropiarse del conocimiento disciplinar (aún no son capaces de emitir juicios críticos) y elaborar la Discusión. Esta suele remitirse a una repetición de los resultados que se han obtenido, sin llegar a generar una verdadera Discusión que confronte sus hallazgos con lo que se plantea en la literatura especializada:

Eso le falta mucho a los alumnos. Durante toda la tesis lo que más les cuesta es aprender la discutir y no repetir otra parte de la tesis en la Discusión (BIO4).

Por último, como se desprende de lo presentado hasta el momento, pese a la reticencia de los docentes por abordar una enseñanza consciente y sistemática que permita desarrollar las habilidades de escritura y argumentación, se destaca su rol como guía y mediador del proceso. Pues aun cuando reconozcan que no poseen el conocimiento metodológico de la escritura en ciencias para hacerlo, recurren a su conocimiento como especialistas para permitir un acercamiento de los estudiantes a las maneras en que se accede y construye conocimiento en la disciplina.

Nos dimos cuenta que también es importante reforzar el área de comunicar la ciencia o el conocimiento científico que ellos pudieran levantar, entre comillas, a través de lo escrito, porque es un ejercicio que es útil, para ellos pensando en que puedan realizar investigaciones (BIO8).

El destacado en la cita anterior enfatiza la relación entre la escritura epistémica y la producción de conocimiento científico en la comunidad.

\subsection{EL CASO DE LA LICENCIATURA EN HISTORIA}

En cuanto al análisis realizado a las seis entrevistas en profundidad a los docentes del programa de Historia que constituyen el corpus de estudio, se relevaron 550 categorías preliminares que fueron agrupadas en $10 \mathrm{CG}$, así como se muestra en el Gráfico 2 que aparece más abajo. Lo primero que es necesario señalar es que ninguna de dichas categorías fue etiquetada como Argumentación; sin embargo, en el discurso de los docentes se reconocen alusiones al tema central de este artículo, aunque de manera tangencial. Por ejemplo, a propósito de la conversación que surge acerca de los tipos de evaluaciones que se realizan en la Licenciatura, los docentes señalan:

La prueba tipo ensayo para la casa es desarrollar un análisis histórico a partir de los conocimientos que ellos tienen, para interpretar esa fuente. Por lo tanto, ellos tienen que argumentar en torno a ese criterio general (HIST4). 
Aplicando el análisis de contenido al segmento anterior, el foco lo constituye el instrumento evaluativo (prueba tipo ensayo para la casa). Así, al momento de realizar un análisis de fuentes, la consecuente interpretación demanda la realización de un ejercicio argumentativo.

De las $550 \mathrm{CP}, 41$ aluden a la argumentación, lo que equivale a 7,65\% de total. Esas $41 \mathrm{CP}$ están incluidas en las CG Propósitos de la escritura, Estrategias metodológicas, Factores que influyen en la escritura, Escritura en Historia y Rol docente. Por ejemplo, la categoría Propósitos de escritura se construyó -en parte- con la CP denominada Escribir para analizar, interpretar y argumentar en la prueba y Escribir para convencer y Rol docente con Valoración positiva de estudiantes que argumentan.

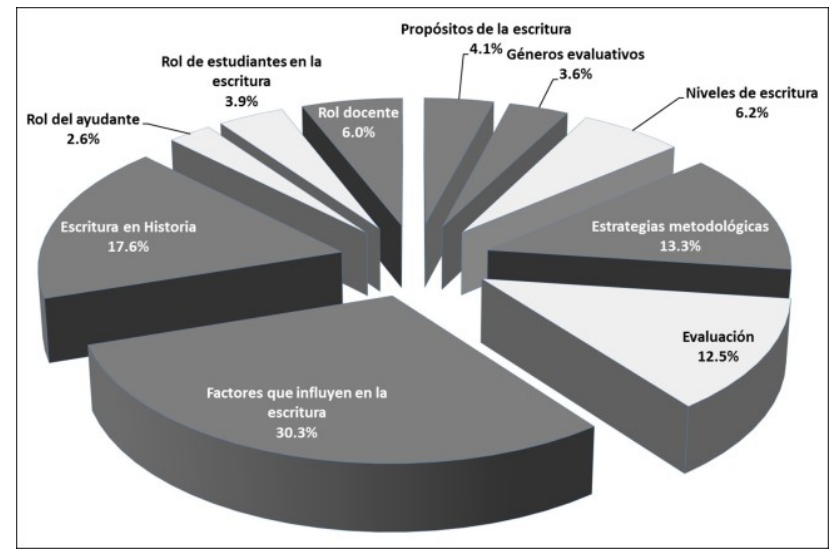

Gráfico 2: Categorías generales de discurso de docentes en Historia

A partir de las CG y así como se realizó con los datos obtenidos a partir del análisis de las RS de los docentes de la comunidad de Biología, se procede a presentar el modelo explicativo construido en Historia, como aparece en la Figura siguiente:



Figura 3: Modelo explicativo de Historia 
$\mathrm{Al}$ contrario de lo que ocurre en Biología, los docentes señalan que no se hacen cargo de problemas que, a su juicio, son heredados del sistema escolar. Además, coinciden en reconocer que, por formación, no estarían capacitados para trabajar el desarrollo de esta habilidad con los estudiantes. Acerca de esto, en un estudio anterior, coincidentemente González y Riquelme ya hacían ver que en esta disciplina:

La escritura se considera el medio esencial a través del cual se transmite la construcción de la historia, sin embargo, no hay una enseñanza directa ni una instancia enfocada en el desarrollo de esta habilidad, aludiendo que debiese venir ya internalizada desde la Enseñanza Media y que no existe el tiempo necesario para realizarla en la Universidad y, por lo tanto, debería ir mejorando con la práctica a lo largo de la Licenciatura (84).

En este sentido, los esfuerzos que se han hecho por abordar su enseñanza son escasos y no tienen continuidad

porque aun cuando, no sé si se puede notar que en los profesores, mejor dicho en algún tipo de profesores, están preocupados de ese tipo de cosas, como dije, de ir enseñando (a escribir), qué se yo, pero no es sistemático, no forma parte de un plan de la carrera, entonces a veces son esfuerzos aislados, no siempre son permanentes y se prueba (HIST5).

A raíz de lo anterior, las estrategias didácticas que se tienden a emplear en las distintas cátedras del programa de Licenciatura están orientadas a colocar un marcado acento en el análisis, la discusión y la crítica, ya sea por medio de dinámicas en clase o mediante lecturas que se encargan a los estudiantes.

Lo que yo trato en el fondo es que la clase no se transforme en una cuestión absolutamente y meramente expositiva donde el profesor prácticamente se endiosa y los estudiantes no participan, no, en este caso mi clase consiste en que uno levanta la mano, opina, o simplemente critica lo que yo digo, porque así también se va construyendo... (HIST3).

A su vez, estas habilidades se ponen de manifiesto en los géneros discursivos que los estudiantes deben producir durante su formación, que se caracterizan por orientarse hacia lo académico-no-científico. En este caso, el foco de estos trabajos también está relacionado con el propósito de justificar, pero, a diferencia de lo que ocurre en Biología (en que se trata de justificar los hallazgos mediante investigaciones), lo que se busca es que los estudiantes justifiquen, frente a su comunidad, que efectivamente han aprendido a lo largo de su formación, como una forma de posicionarse frente a sus pares. Por lo que este tipo de ejercicios adquieren un cariz netamente evaluativo.

Por ejemplo, la argumentación, la argumentación cómo la verificas tú, no por la bibliografía, sino más bien por el uso de aparato de notas. Qué leyó, cómo lo leyó, qué citas seleccionó, es la cita pertinente, el autor es pertinente o no es pertinente, 
el mismo autor se repite diez veces en Ibídem o en realidad se contrasta con otro. Y bueno, generalmente el juicio final... (HIST2).

Es admisible resaltar que la justificación de ideas que se realiza en el discurso es mucho más personal, por lo que se reconoce como una característica de la escritura en Historia la idea de inscribirse en el discurso, es decir, escribir desde un yo (yo justifico, yo valoro, yo argumento), otorgándole un valor mucho más subjetivo al texto.

Pese que, en cierta manera, las estrategias que emplean los docentes permiten que los estudiantes puedan superar los retos que se plantean en el programa, existen ciertos factores que intervendrían en el desarrollo de la escritura disciplinar y, por consiguiente, en el ejercicio argumentativo. Quizá los más relevantes son la memorización, que dificulta la emisión de opiniones o juicios críticos; el no poder inscribirse efectivamente en el discurso, sino que refugiarse en lo que otros señalan (o hablar impersonalmente).

Entonces los estudiantes tienen la tendencia a no jugarse en el relato, entonces te pueden colocar, ponte tú, la guerra de la independencia...el concepto de guerra e independencia (...) al final, te dice todo, claro, todo lo que se ha escrito, pero no está él en el relato (HIST5).

Finalmente, como ha quedado en evidencia, el trabajo que se realiza en Historia está ligado estrechamente con la necesidad de desarrollar habilidades que les permitan a los estudiantes abordar los problemas que se les plantean en el programa. En todo momento, el énfasis está puesto en la evaluación de sus conocimientos y competencias, pero siempre referidos a un contexto académico de formación disciplinar.

\section{COMENTARIOS FINALES}

Luego de la construcción de sendos modelos explicativos para cada una de las disciplinas, se señala que en el discurso de los docentes de ambas se reconoce la importancia de la argumentación, con algunas diferencias. En Biología se releva la argumentación con el peso de una categoría que la posiciona con fuerza al interior de la comunidad, esto es, el 19,96\% de los segmentos de las transcripciones de las entrevistas a los docentes corresponde a dicha categoría, ya sea de manera directa o tangencial. En Historia ocurre una situación diferente: la argumentación no se releva como categoría, aunque se reconoce su aparición de manera indirecta y el porcentaje de segmentos dedicados a este tema alcanza a 7,65\%. Pudiera pensarse a priori que Historia, por ser una disciplina de las humanidades, evidencia una relación más marcada con la argumentación que Biología, por su objeto de estudio y la naturaleza de su conocimiento. Sin embargo, esta idea previa no se confirma con el trabajo de levantamiento de datos. Si se considera que los objetivos de la educación universitaria es el cuestionamiento del conocimiento establecido y la generación de nuevo conocimiento, resulta curioso que a la argumentación no se le reconozca más 
importancia, sobre todo porque esta incide en el desarrollo de las habilidades de pensamiento crítico y en el desarrollo de la función epistémica de la escritura.

Asimismo, el análisis de los segmentos textuales demuestra que en las comunidades particulares que se analizaron, la argumentación es un tema representado socialmente de distinto modo por los miembros de la comunidad. Mientras Biología asume como eje central la objetividad en el discurso, por lo que la argumentación se inscribe como una forma de respaldo y de validación de los resultados obtenidos y del modo de hacer ciencia; en Historia, el foco está concentrado en la implicación del sujeto en el discurso y su posicionamiento personal frente a los otros, por lo que la argumentación está al servicio de respaldar sus ideas ante los demás. Es así como en Biología la argumentación coadyuva al posicionamiento en la disciplina; en cambio, en Historia esta se configura en función de la comunidad.

En Biología, la argumentación se reconoce como una habilidad fundamental para el desarrollo del pensamiento científico que implica tanto justificar los propios resultados obtenidos como cuestionar los hallazgos de otros miembros de la comunidad. La relación entre argumentación, justificación, pensamiento científico y escritura -como divulgación o construcción del conocimiento científico- aparece claramente explicitada a partir de las RS de los docentes. Se reconoce asimismo la dificultad que evidencian los estudiantes para poner en ejecución este proceso discursivo y las estrategias compensatorias que se utilizan para propiciar la inserción de los estudiantes en la comunidad. Situación similar ocurre en Historia frente a la relación que se establece entre argumentación, pensamiento histórico y escritura, con la diferencia de que los docentes asumen que no se hacen cargo de enseñar la escritura con las convenciones de la comunidad.

En Historia, la argumentación se concibe como una actividad permanente, que permea todas las actividades. Se configura bajo el amparo de la acreditación del conocimiento, es decir, todos los instrumentos de evaluación consideran preguntas que implican argumentar, ya sea pruebas que se desarrollan en clases, domiciliarias, controles de lectura, informes, pruebas tipo ensayo, etc. En cambio, en Biología la argumentación se concentra en la sección de discusión de los datos en los trabajos de investigación, en los informes de salida a terreno o en los de laboratorio. Mediante las RS de los docentes se puede establecer que el foco principal para el desarrollo de la argumentación es la variedad de géneros académicos de carácter acreditativo que circulan en las distintas comunidades. Por tanto, el esfuerzo de enculturación de cada comunidad académica podría centrarse en la comprensión y producción de los géneros que circulan en cada comunidad, desde un enfoque procesual y estratégico, con una función marcadamente epistémica, con un enfoque centrado en la metacognición y en algunos elementos metalingüísticos, con un marcado énfasis en las habilidades que posibilitan el pensamiento crítico, analítico, reflexivo y sintético y en un esfuerzo coordinado entre el especialista en Lenguaje y el especialista disciplinar. 
El rol de la argumentación en el pensamiento crítico y en la escritura epistémica

Pontificia Universidad Católica de Valparaíso* Avenida El Bosque 1290 Campus MT Brown de Ariztía, Viña del Mar (Chile) alejandro.cordova@pucv.cl

Pontificia Universidad Católica de Valparaíso** Avenida El Bosque 1290 Campus MT Brown de Ariztía, Viña del Mar (Chile) marisol.velasquez@pucv.cl

Pontificia Universidad Católica de Valparaíso *** Avenida El Bosque 1290 Campus MT Brown de Ariztía, Viña del Mar (Chile) lis.cep@gmail.com

\section{OBRAS CITADAS}

Arenas, Lisbeth y María Gabriela Olivares. "Criterios de evaluación de los informes de investigación en la Licenciatura en Bioquímica". Comunidades académicas y culturas escritas. Construcciones discursivas desde las Ciencias y las Humanidades, En Juana Marinkovich, Marisol Velásquez, Alejandro Córdova (Eds.). Valparaíso: Ediciones Universitarias de Valparaíso, 2012: 119-125.

Carlino, Paula. "Alfabetización académica diez años después", Revista Mexicana de Investigación Educativa 18/57 (2013): 355-381.

Carlino, Paula, Patricia Iglesias e Irene Laxalt. "Concepciones y prácticas declaradas de profesores terciarios en torno al leer y escribir en las asignaturas", Revista de Docencia Universitaria 11/1 (2013): 105-136.

Córdova, Alejandro y Marisol Velásquez. "Licenciatura en Bioquímica: Influencia del método científico en la escritura académica". Comunidades académicas y culturas escritas. Construcciones discursivas desde las Ciencias y las Humanidades. Juana Marinkovich, Marisol Velásquez, Alejandro Córdova (Eds.). Valparaíso: Ediciones Universitarias de Valparaíso, 2012: 71-82.

Fernández, Lorena. La enseñanza de la escritura académica en las universidades del área Metropolitana de Buenos Aires (tesis de maestría). Buenos Aires: Universidad de San Andrés, 2010.

Fisher, Alec y Michael Scriven. Critical Thinking. Its Definition and Assessment. Cambrige: Edgepress and Centre for Research in Critical Thinking, University of East Anglia, 1997.

González, Felipe y Tamara Riquelme. "Licenciatura en Historia: Construyendo la historia a través de la escritura". Comunidades académicas y culturas escritas. Construcciones discursivas desde las Ciencias y las Humanidades. Juana Marinkovich, Marisol Velásquez, Alejandro Córdova (Eds.). Valparaíso: Ediciones Universitarias de Valparaíso, 2012: 83-93.

Gorodokin, Catalina y Myriam Mercau. "Nosotros argumentamos... ¿y vos? Relato de una experiencia didáctica”, Revista Iberoamericana de Educación 34/5 (2005): 1-10. 
Holsti, Ole. "Content analysis". The Handbook of social Psychology. Gardner Lindzey y Elliot. Aronson (Eds.), Reading, MA: Addison-Wesley, 1968: 596-692.

Ilardo, Joseph. Speaking persuasively. New York, NY: Macmillan Publishing, 1981.

Jodelet, Denise. "Représentations Sociales: un domaine en expansion". Les Représentations Sociales. D. Jodelet (Ed.). Paris: PUF, 1989: 31-61.

Marinkovich, Juana, Marisol Velásquez y María Gabriela Olivares. "Niveles de alfabetización en escritura académica: de la reproducción a la transformación del conocimiento disciplinar". Estudios del discurso en América Latina. Homenaje a Anamaría Harvey. Neyla Pardo, Denize García, Teresa Oteíza y María Asqueta, (Comp.) Bogotá: ALED, 2013:129-149.

Moscovici, Serge. "On social Representations". Social Cognition. Perspectives in everyday life. J.P. Forgas (Ed.). London: Academic Press, 1981: 181-209.

Mota de Cabrera, Carmen. "Desarrollo del pensamiento crítico a través del discurso argumentativo: Una experiencia pedagógica en un curso de lectura y escritura". Entre Lenguas. Universidad de Los Andes: Mérida, 2010:15/11-23.

Navarro, Federico. "Géneros discursivos e ingreso a las culturas disciplinares. Aportes para una didáctica de la lectura y la escritura en educación superior". Manual de escritura para carreras de humanidades. Federico Navarro (Coord.). Buenos Aires: eFFL, 2014: 29-52.

Otis, William. Speaking to inform and persuade. New York, NY: Macmillan Publishing, 1982.

Padilla, Constanza. "Iniciación a las prácticas académicas: experiencia en un nuevo espacio curricular de la carrera de Letras (UNT)". Actas de I Jornadas interdisciplinarias "Lenguas, Identidad e Ideologías", Tucumán, UNT, 2007.

_ "Taller de comprensión y producción textual: un espacio para leer y escribir géneros académicos". Actas del I Congreso Nacional de Estudios Comparados en Educación, Buenos Aires, SAECE, 2005.

__ "Producción de discursos argumentativos en estudiantes universitarios". En Actas del V Congreso de Lingüistica General, 3 (2004): 2193-2202.

Padilla, Constanza, Silvina Douglas y Esther López. "Competencias argumentativas en la alfabetización académica", Revista Innovación Educativa 4 (2010): 2-12.

Paul, Richard y Linda Elder. La mini-guía para el pensamiento crítico: conceptos y herramientas, 2003. Rescatado el 15 de febrero de 2013. Disponible en:

https://www.criticalthinking.org/resources/PDF/SP-ConceptsandTools.pdf.

Perelman, Chaïm y Lucie Olbrechts-Tyteca. Tratado de la Argumentación. La Nueva Retórica. Gredos: Madrid, 1969.

Porta, Luis y Miriam Silva. La investigación cualitativa: El análisis de contenido en la investigación educativa. Mar del Plata. Rescatado el 4 agosto de 2003. Disponible en: http:/www.uccor.edu.ar/paginas/REDUC/porta.pdf.

Ruiz, José. Metodología de la investigación cualitativa. Bilbao: Universidad de Deusto, 2007. 
Serrano, Stella y José Villalobos. Niveles discursivos del texto argumentativo escrito por estudiantes de formación docente. Universidad de Los Andes: Mérida, 2008.

— La argumentación discursiva escrita. Teoría y práctica. Universidad de Los Andes: Mérida, 2006.

Teberosky, Anna. "El texto académico". Coord. Montserrat Castelló. Escribir y comunicarse en contextos científicos y académicos: Conocimientos y estrategias. Barcelona: GRAÓ, 2007: 17-46.

Toulmin, Stephen. The Uses of Argument. Cambridge University Press: Cambridge, 1958.

Van Eemeren, Frans, Rob Grootendorst y Francisca Snoeck. Fundamentals of Argumentation Theory: A Handbook of Historical Backgrounds and Contemporary Developments. Lawrence Erlbaum: Mahwah, 1996. 